\title{
A Novel Protocol for Detection and Optimization of Overlapping Coverage in Wireless Sensor Networks
}

\author{
Vipul Narayan, A.K. Daniel
}

\begin{abstract}
Wireless sensor network (WSN) is an emerging area in which numbers of sensor nodes are deployed in two ways random and deterministic for data gathering. WSNs have expedited human life in diverse emerging fields: military, agriculture, structural health, perimeter access control, forest fire detection. A physical stimulus such as pressure, sound, light act as an environmental parameter on which the system is design to monitor and detect it for controlling the coverage area for assigned task. The nodes are deployed in the random manner in the given area for gathering the information and they may be overlapped so that the total area may not be covered. The proposed protocol uses radius and the residual energy as a function to increase the total coverage area so that the whole coverage area may be achieved. It also increases the life time of the network using Sleep and Wakeup protocol. Therefore the overall life time of the network increases.
\end{abstract}

Catchphrases: Sensor, Residual energy, Coverage area,

\section{I.INTRODUCTION}

WSN is often outlined as a set of sensor nodes that convert the sensed information from the environment like temperature, pressure, speed, humidity. Signals measured by different types of transducers are used to convert these signals into electrical signals and then converted into digital signals for processing, analyzing, storing and transmitting the information to the base station. Sensors are low power devices that communicate within the distance (range) through radio frequency. Sensor nodes consist of the following Unit, Processing Unit and Storage Unit [1].The sensing unit sensed the information and preprocess it in the form of data. Theses data are stored and processed to generate the signals to be transmitted to the base station through multi-hop system. Many important application of WSN plays an important role in every aspect of life. It is used for monitoring and tracking of objects in various fields like air pollution, forest fire detection, wine quality enhancing, offspring care, sports men care, structural health, radiation levels, perimeter access control, smart phone detection, electromagnetic levels, traffic congestion, water quality, smart parking, item location, vehicle auto diagnosis,

water leakages, intelligent shopping, smart parking, item location, vehicle Figure No.1 Components of auto-

Revised Manuscript Received on August 14, 2019.

Vipul Narayan, Department of Computer Science and Engineering, Madan Mohan Malaviya University of Technology Gorakhpur, U.P, India (Email: vipulupsainian2470@gmail.com)

A.K. Daniel, Department of Computer Science and Engineering, Madan Mohan Malaviya University of Technology Gorakhpur, U.P, India (Email: danielak@rediffmail.com) Network lifetime.

diagnosis, water leakages, intelligent shopping, smart lighting, smart roadways. Section I describes to WSN and its various important components. Section II discusses the literature review done by various authors. Section III discusses the motivation towards wireless sensor network, characteristic of wireless communication, their applications as well as important challenges and issues. Section IV describes a new protocol for covering total area by ' $n$ ' nodes distribution and having less number of overlapping nodes and in Section $\mathrm{V}$ includes the conclusion.

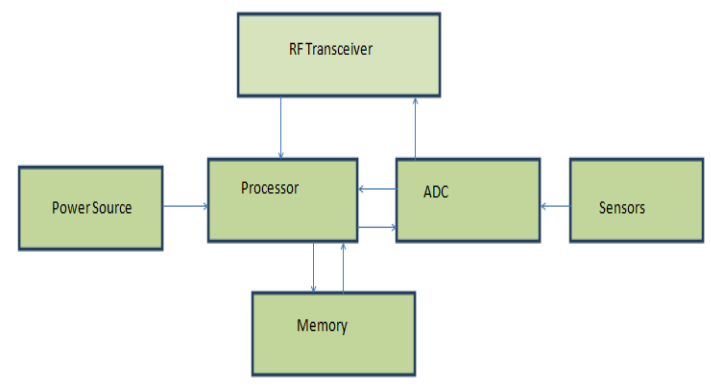

Figure No.1. Components of WSN

\section{LITERATURE SURVEY}

Chi-Fu Huang et al. [1] have assumed the coverage problem as a decision problem whose aim is to check whether every target in the field of interest is covered by at least $\mathrm{k}$ (predefined value) sensors or not. The sensing range is further classified as unit disks (The k-UC Problem) and non-unit disks (The k-NC Problem). Former has the same sensing range of all the sensors that are $\mathrm{S}_{\mathrm{r} 1}=\mathrm{S}_{\mathrm{r} 2}=\ldots=\mathrm{S}_{\mathrm{rn}}$ where $S_{r}$ is the sensing range of the sensor $(S)$ and the later has a different sensing range of all the sensors.

My T. Thai et al. [2] have presented an analysis and overview of the solutions proposed for the coverage problems and have components known as Sensing Unit, Communication also done the survey on the state-of-the-art coverage formulation. The coverage problem also affects the QoS of the network. In the paper authors have discussed the various coverage problems definition like $\mathrm{k}$-Coverage, Maximum Lifetime Coverage, Minimum Coverage Breach, Connected coverage and Connected k-Coverage.

Yao et al. [3] explained the major problem in sensor network: the coverage and connectivity difficulty for providing better services in terms of quality and reliability.
Blue Eyes Intelligence Engineering \& Sciences Publication 
Coverage problem is defined in terms of how long and how well the network is able to monitor the physical environment. Coverage problem is broadly classified into two categories: Area Coverage and Target Coverage. In this paper authors have discussed the various techniques for solving the coverage problems which also increases the network lifetime, such as Grid-based, Force based and Computational geometry based. The different existing techniques for solving coverage problem are classified in terms of node types, coverage types, energy constraints, deployment, three-dimensional coverage and centralized and distributed algorithm.

Sangeetha S et al. [4] have investigated all the coverage problem and addressed the various coverage approaches like Probabilistic sensing, coverage with connectivity, Disjoint sets, Maximal support path (best coverage) and Maximal breach path (worst coverage). The existing techniques for solving coverage problem are classified in terms of node types, coverage types, energy constraints, deployment, three-dimensional coverage and centralized and distributed algorithm.

S. Mini et al. [5] have addressed M-connected target coverage problem for $\mathrm{k}$-coverage, Q-coverage and simple coverage. M-connected target coverage in WSN is defined as a network where all the sensor nodes are connected with at least ' $M$ ' other sensor nodes. The proposed work of $M$ connectivity is inspired by Low-Energy Adaptive Clustering Hierarchy (LEACH) model and has a better result as compared to Communication Weighted Greedy Cover (CWGC).

Daniel et al. [6] discussed the important challenging problems in the wireless sensor network. They focused on different types of hole occurrence and detection problems in the network. They also gave the technique to solve the coverage hole problem. For the repairing of holes problem in the areas of logical and semantic holes is rarely used. In future they will work on the detection, avoidance, prevention and healing of the holes.

Daniel et al. [7] discussed the evolution of sensor nodes, challenging problems and different types of wireless sensor network and types of coverage. They have discussed different approaches to solve the problems and found that research is required for achieving coverage area.

Daniel et al. [8] discussed the EECP based trust model that supports trusted communication, trusted energy, trusted data and node contributions. The performance improvement of the network life cycle by a factor of 12.5 unit times that of TBCA approach. The overall system improves the performance in terms of data reliability, coverage level, network life time and energy saving.

Daniel et al. [9] stated that WSN has resource constrained property and the aim is to maximize the lifetime of network and coverage level.The various coverage models are discussed for the sensing ability in terms of quantity and quality. Quantity is measured in terms of the noise factor, signal power, Antenna Gain, Bandwidth of the signal and Antenna, received signal power and signal power is discussed. Quality is measured in terms of node coverage in the target area. It is found that Probabilistic coverage achieves better simulation results compares to other models in terms of optimal values as 0.1 and distance is achieved 24.5 units.

Sing et al. [10] stated that the major issues in the wireless sensor network are the coverage and connectivity problem for providing better QoS. Coverage problem is defined in terms of how long and how well the network is able to monitor the physical environment. Coverage problem is broadly classified into two sections: Area coverage and Target coverage. In this paper authors have studied the various approaches for solving coverage problems and to increase the network lifetime such as Gridbased, Force based and Computational geometry based.

\section{MOTIVATION}

In WSN the sensor nodes have limited battery power so our goal is to develop and deploy a sensor node in effective way such that life time of the sensor network increases. The deployment must be in such a way that it minimizes the communication overhead and coverage problem. Several techniques have been proposed in the literature review to tackle the limitations of the WSN such as less memory and computation requirement to reduce the energy requirement. Important features of WSN are data collection, maintenance, flexibility, lifetime, diverse application, unique network topology, unique traffic constraints and severe resource constraints. Important characteristic of WSN are distributed sensing property, low power consumption, short range communication in convenient and reliable manner, flow control and error control mechanism for error detection and error correction. The most important research issue and challenges in WSN is to yield optimal coverage. This ensures that the target should lies within the sensing area of the sensor nodes. There are various challenges and issues in deployment of sensor nodes such as QoS, Harsh Environment Energy, Security, Localization, Data aggregation and data dissemination are major issue in sensor nodes, the dimension of the RoI, continuous connectivity in the network and enhancement of the network lifetime by using a minimum number of sensor nodes. Random deployment, sensing and detection model is also the most important challenges and issues in WSNs [11] [12][13].

\section{THE PROPOSED PROTOCOL FOR TOTAL AREA COVERED \& RESULTS}

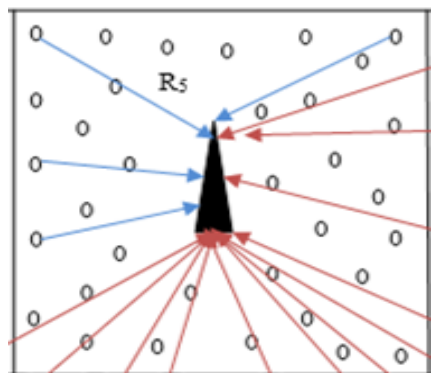

Figure No.2. Deployment of nodes in the coverage area 
The sensing region is the feasible distance from the source node to the RoI where the target must be covered by the sensor.

\section{Case 1:}

(For Target or Point Coverage) - Consider sensor $\mathrm{S}_{\mathrm{i}}$ located in position $\left(\mathrm{x}_{\mathrm{i}}, \mathrm{y}_{\mathrm{i}}\right)$ with radius $\mathrm{S}_{\mathrm{r}}$ and target or point $(\mathrm{P})$ is located in position $\left(\mathrm{x}_{\mathrm{p}}, \mathrm{y}_{\mathrm{p}}\right)$ respectively. Then

$$
\mathrm{d}\left(\mathrm{P}, S_{\mathrm{i}}\right)=\sqrt{\left|\mathrm{x}_{\mathrm{p}}-\mathrm{x}_{\mathrm{i}}\right|^{2}+\left|\mathrm{y}_{\mathrm{p}}-\mathrm{y}_{\mathrm{i}}\right|^{2}}
$$

Where the Euclidean distance between the point $(\mathrm{P})$ and the sensor node can be represented by $\mathrm{d}\left(\mathrm{P}, \mathrm{S}_{\mathrm{i}}\right)$ and is said to be covered by the sensor $\left(\mathrm{S}_{\mathrm{i}}\right)$ if and only if $\mathrm{d}\left(\mathrm{P}, \mathrm{S}_{\mathrm{i}}\right) \leq \mathrm{S}_{\mathrm{r}}$

\section{Case 2:}

(For Area Coverage) - Consider an area (A) which is said to be covered by the sensor $\left(\mathrm{S}_{\mathrm{i}}\right)$ if and only if all the targets or points $(\mathrm{P})$ in the area $(\mathrm{A})$ is covered by the sensor $\left(\mathrm{S}_{\mathrm{i}}\right)$.

Deployments of nodes are the important issue of coverage is to yield optimum coverage. The coverage is the most important issue of the sensor node deployment. The overlapping of nodes in the coverage area is to reduced as possible so that the node energy may be minimized by using Sleep or Wakeup issue.

The rate of change of Area coverage is equivalent to the rate of change of overlapped with node coverage.

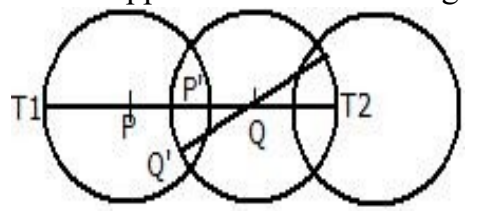

Figure No. 3. Total area covered

Total Area Covered $=$ PP $`$ QQ

$$
\begin{aligned}
& =\mathrm{PP}^{\prime}+\mathrm{QQ}^{\prime}-\mathrm{P}^{\prime} \mathrm{Q}^{\prime} \\
& =\mathrm{r}+\mathrm{r}-\Delta
\end{aligned}
$$

Total Area Covered $(\mathrm{A})=2 \mathrm{r}-\Delta$

$$
\mathrm{A}=2 \mathrm{r}-\Delta \quad \begin{aligned}
& \frac{\delta A}{\delta \Delta}=\frac{\delta \mathrm{A}}{\delta \mathrm{r}} *\left(\frac{\delta \mathrm{r}}{\delta \Delta}\right) \\
& \frac{\delta A}{\delta r}=2-\left(\frac{\delta \Delta}{\delta \mathrm{r}}\right) \\
& \frac{\delta^{2} A}{\delta r^{2}}=0-\frac{\delta^{2} \Delta}{\delta r^{2}} \\
& \frac{\delta^{2} A}{\delta r^{2}}=-\left(\frac{\delta^{2} \Delta}{\delta r^{2}}\right)
\end{aligned}
$$

Since the second derivative of the above function is a negative value hence we get local maxima for the mentioned function. e.g.

$$
\begin{gathered}
\frac{\delta A}{\delta r} \max \\
f(u, v)=\frac{\delta u}{\delta x} *\left(\frac{\delta x}{\delta v}\right)
\end{gathered}
$$

Maximum Transmission Range $=\mathrm{T}_{1} \mathrm{P}+\mathrm{PP}^{`}+\mathrm{P}^{`} \mathrm{Q}+\mathrm{QT}_{2}$

$$
=\mathrm{r}+\mathrm{r}-\mathrm{Q}_{1} \mathrm{P}_{1}+\mathrm{r}+\mathrm{r}
$$

$=2 \mathrm{r}+\mathrm{r}+\mathrm{r}-\Delta$

$=(4 \mathrm{r}-\Delta)(\mathrm{n}-2)$

$=(\mathrm{n}-1)(4 \mathrm{r}-\Delta)$

$=4 \mathrm{r}(\mathrm{n}-1)-\Delta(\mathrm{n}-1)$

$\mathrm{F}(\mathrm{r})=4 \mathrm{r}(\mathrm{n}-1)-\Delta(\mathrm{n}-1)$

$$
=(\mathrm{n}-1)(4 \mathrm{r}-\Delta)
$$

$\mathrm{A} \quad=2 \mathrm{R}-\Delta$

\section{$\Delta=2 \mathrm{R}+\mathrm{A}$}

Putting the value of $\Delta$ in equation (1) we get

$\mathrm{F}=(\mathrm{n}-1)[4 \mathrm{r}-2 \mathrm{r}+\mathrm{A}]$

$\mathrm{F}=(\mathrm{n}-1)[2 \mathrm{r}+\mathrm{A}]=\mathrm{F}(\mathrm{r}, \mathrm{A})$

$$
\begin{aligned}
& \frac{\delta F}{\delta r}=(n-1)(2) \\
& \frac{\delta F}{\delta A}=(n-1)(1)
\end{aligned}
$$

$\mathrm{F}(\mathrm{x}, \mathrm{y})$

$$
\begin{aligned}
& \frac{\delta F}{\delta x}=0 \frac{\delta F}{\delta y} \rightarrow 0 \\
& \frac{\delta^{2} F}{\delta r^{2}}=0 \\
& \frac{\delta^{2} F}{\delta A^{2}}=0
\end{aligned}
$$

$\mathrm{F}(\mathrm{x}, \mathrm{y})$

$$
\frac{\delta^{2} F}{\delta r \delta A}=0
$$

$$
\frac{\delta F}{\delta x}=0 \frac{\delta F}{\delta y} \rightarrow 0
$$

We know that Maxima and Minima for two variables $\mathrm{F}(\mathrm{x}, \mathrm{y})$ can be represented as:

$$
\mathrm{D}=\mathrm{rt}-\mathrm{s}^{2}=\frac{\delta^{2} F}{\delta x^{2}} \cdot \frac{\delta^{2} \mathrm{~F}}{\delta y^{2}}-\frac{\delta^{2} F}{\delta x \delta y}
$$

Where

$$
\mathrm{r}=\frac{\delta^{2} \mathrm{~F}}{\delta \mathrm{x}^{2}}, \mathrm{t}=\frac{\delta^{2} \mathrm{~F}}{\delta \mathrm{y}^{2}}, \mathrm{~s}^{2}=\frac{\delta^{2} \mathrm{~F}}{\delta \mathrm{x} \delta \mathrm{y}}
$$

Similarly for $\mathrm{F}(\mathrm{r}, \mathrm{A})$ we have $r=\frac{\delta^{2} \mathrm{~F}}{\delta \mathrm{r}^{2}}, \mathrm{t}=\frac{\delta^{2} \mathrm{~F}}{\delta \mathrm{A}^{2}}, \mathrm{~s}^{2}=\frac{\delta^{2} \mathrm{~F}}{\delta \mathrm{r} \delta \mathrm{A}}$

Which gives the result:

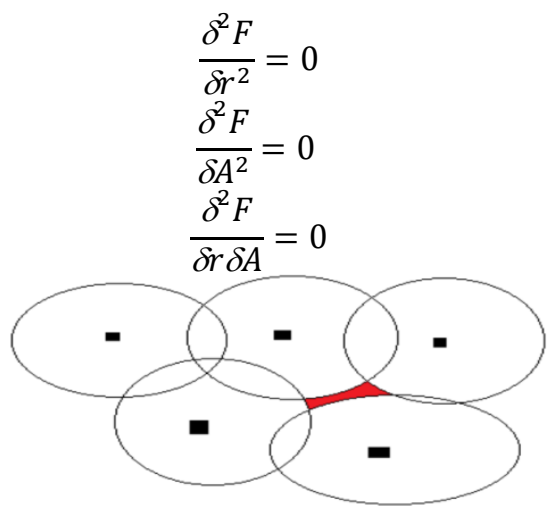

Figure No. 4. Coverage Hole

Since the First derivative is constant therefore the second derivative will produce result zero.

$\mathrm{F}(\mathrm{r}, \mathrm{A})$ is a linear function so it becomes a free variable. Both $\mathrm{F}(\mathrm{r}, \mathrm{A})$ are Independent to each other.

The nodes are overlapped in the given region by more than one node i.e. the nodes are intersect each other. Let there are 2 nodes then intersect is 1 , if there are three nodes then intersect is 4 and so on.

If we have ' $n$ ' then the intersection will be greater than 'n'nodes. 


\section{Algorithm \\ Input: $\mathrm{N}, \mathrm{r}_{\mathrm{i}}, \mathrm{IR}, \mathrm{IL}$}

Initialization: $\quad \mathrm{CR}=$ Current radius, $\mathrm{IR}=$ Initial radius, $\mathrm{IL}=$ Initial lifetime, $\mathrm{G}=$ Nearest neighbor node, $\mathrm{M}=$ Reference Node, $\mathrm{R}=$ Redundant node, $\mathrm{N}=$ Number of nodes, $\mathrm{D}=$ Duplicate nodes, $\mathrm{I}=$ Intersect Nodes, $\mathrm{C}=$ Common length set $(\mathrm{c} 1, \mathrm{c} 2 \ldots \ldots . \mathrm{ck}), \mathrm{D}_{\mathrm{N}=}$ Duplicate node.

Output: Minimum number of sensors.

Nodes are in sleep state and at anytime only one is active.

Rule1: The nodes which are overlapped may only be Turnoff.

Rule2: If the node is not covering any other node and is also in Sleep state then it should move to overlapped.

Step 1: For all nodes ' $\mathrm{N}$ ' in the network, calculate

if $\mathrm{D} \leq 0.3 * \mathrm{r}_{\mathrm{i}}$ of $\mathrm{M}$

then Turn-on (M) and Turnoff (R).

Step 2:For all nodes ' $\mathrm{N}$ ' in the network

if $\mathrm{D} \leq 130 \%$ of $\mathrm{M}$ and $\mathrm{I} \geq 2$

then Turnoff (M).

Step 3: For all 'I' calculate common coverage area by

$\mathrm{r}_{\mathrm{i}} \leftarrow \mathrm{r}_{\mathrm{i}-}$ (common length - set) $)_{\text {min. }}$

Step 4: For all Nodes ' $N$ ' in the network

if $r_{i} \leq \mathrm{IR} * 0.3$

then Turnoff $(\mathrm{N})$.

Step 5: For all Nodes ' $N$ ' in the network

if $\mathrm{D} \leq \mathrm{r}_{\mathrm{i}}$ of $\mathrm{D}_{\mathrm{N}}$

then Turnoff $(\mathrm{N})$.

Step 6: For all Nodes ' $\mathrm{N}$ ' in the network, calculate by $\mathrm{r}_{\mathrm{i}} \leftarrow \mathrm{c}+(\mathrm{IR} * 0.25)$

Step 7: For all Nodes ' $\mathrm{N}$ ' in the network, $\mathrm{r}_{\mathrm{i}} \leftarrow \mathrm{r}_{\mathrm{i}}+1$ upto Threshold value which is $25 \%$ of IR until they intersect each other.

Step 8: For all Nodes ' $N$ ' in the network,

Lifetime $\leftarrow \mathrm{r}_{\mathrm{i}} * 100 / 10 \leftarrow 10 \mathrm{r}_{\mathrm{i}}$

lifetime $\leftarrow$ lifetime- 1

If lifetime $\leftarrow 0$

Switch new node

\section{ANALYSIS OF THE COVERGAE MODEL}

Considering a network of 10 nodes are deployments randomly at different position in the given $10^{*} 10 \mathrm{~m}^{2}$ area. The node positions are computed and find the overlapping nodes. The simulation is done in Matlab17 following responses are produced for analyses are as follows.

Table No. 1 Coverage range of each node from other nodes

\begin{tabular}{|c|c|c|c|c|c|c|c|c|c|c|}
\hline \multirow{2}{*}{$\begin{array}{l}\text { Nodes/ } \\
\text { Distance }\end{array}$} & \multirow[b]{2}{*}{$\mathrm{Nl}$} & \multirow[b]{2}{*}{$\mathrm{N} 2$} & \multirow[b]{2}{*}{$\mathrm{N} 3$} & \multirow[b]{2}{*}{$\mathrm{N} 4$} & \multirow[b]{2}{*}{ N5 } & \multirow[b]{2}{*}{$\mathrm{N} 6$} & \multirow[b]{2}{*}{$\mathrm{N} 7$} & \multirow[b]{2}{*}{ N8 } & \multirow[b]{2}{*}{ N9 } & \multirow[b]{2}{*}{$\mathrm{N} 10$} \\
\hline & & & & & & & & & & \\
\hline $\mathrm{Nl}$ & 0 & 1.35 & 7.16 & 3.16 & 4.4 & 8.74 & 8.05 & 7.5 & 8.13 & 9.12 \\
\hline $\mathrm{N} 2$ & 1.35 & 0 & 7.85 & 2 & 4.06 & 9.02 & 8.02 & 6.99 & 7.02 & 8.02 \\
\hline $\mathrm{N3}$ & 7.16 & 7.85 & 0 & 7.92 & 5.43 & 3.01 & 4.28 & 6.53 & 10.25 & 10.92 \\
\hline $\mathrm{N} 4$ & 3.16 & 2 & 7.92 & 0 & 2.98 & 8.39 & 7.01 & 5.42 & 5.02 & 6.02 \\
\hline N5 & 4.4 & 4.06 & 5.43 & 2.98 & 0 & 5.43 & 4.06 & 7 & 5.16 & 5.03 \\
\hline N6 & 8.74 & 9.02 & 3.01 & 8.39 & 5.43 & 0 & 2.07 & 4.92 & 3.16 & 9.55 \\
\hline $\mathrm{N7}$ & 8.05 & 8.02 & 4.28 & 7.01 & 4.06 & 2.96 & 0 & 2.86 & 7.08 & 7.49 \\
\hline N8 & 7.5 & 6.99 & 6.53 & 5.42 & 3.12 & 4.92 & 2.86 & 0 & 5.56 & 4.63 \\
\hline N9 & 8.44 & 7.02 & 10.25 & 5.02 & 5.16 & 9.11 & 9.79 & 4.23 & 0 & 1 \\
\hline $\mathrm{N} 10$ & 9.12 & 8.02 & 10.92 & 6.02 & 6.01 & 9.55 & \begin{tabular}{|l}
7.49 \\
\end{tabular} & 4.63 & 1 & 0 \\
\hline
\end{tabular}

The node radius sums of two nodes are less than the sum of radius then they are overlapped.
Table No. 2 The nodes Position of overlapped Nodes

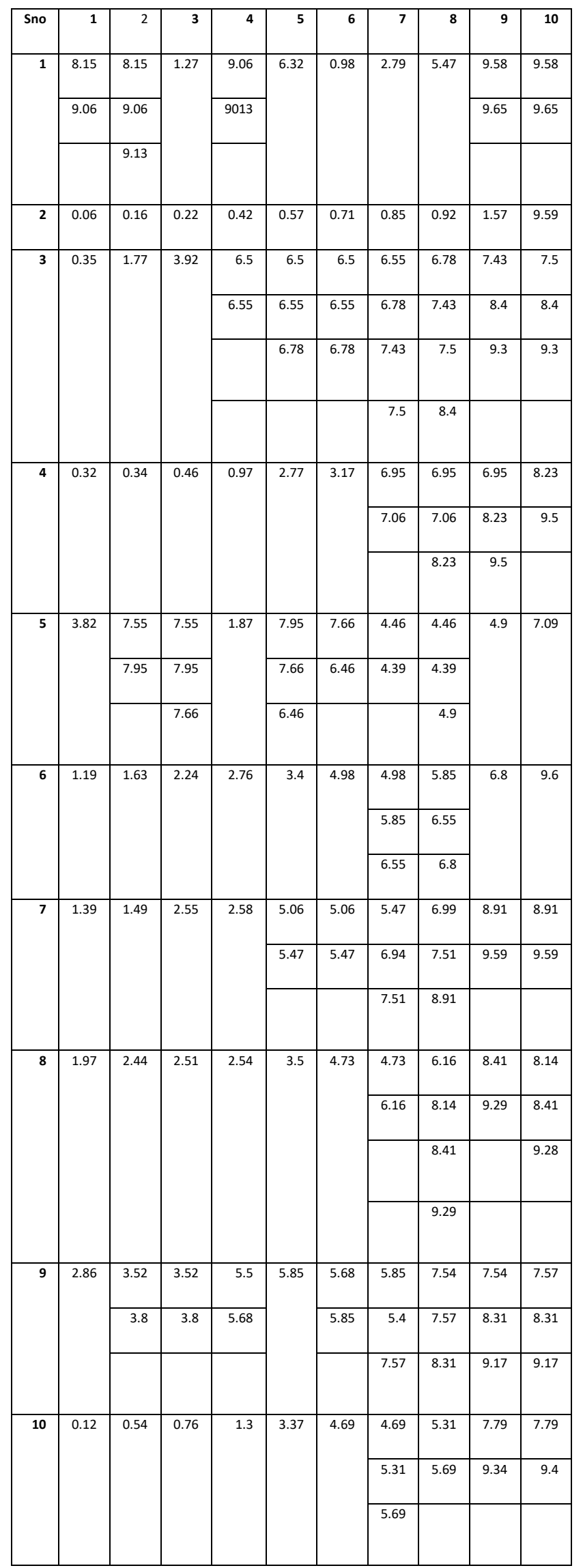




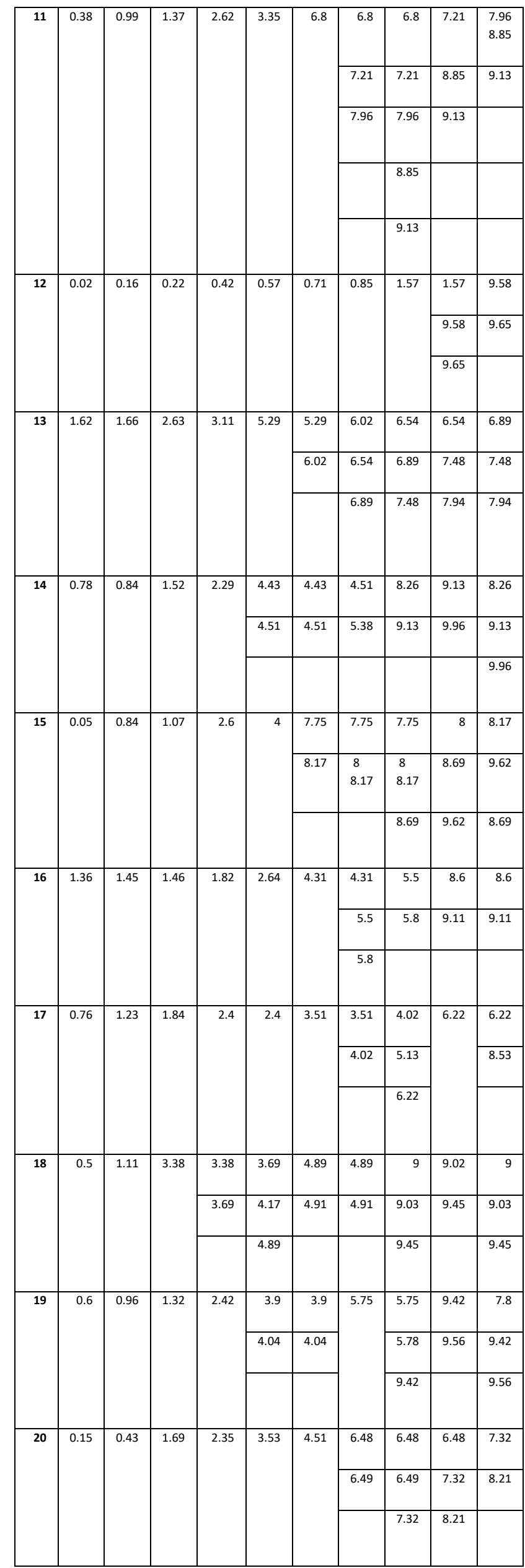

Table 1, Table 2 and Table 3 shows that the overlapping of nodes are after 5 node (s) position i.e. node $6,7,8,9,10$ are overlapped. The overall overlapping is less than $50 \%$.

Table No. 3 No of overlapping nodes found in 20 rounds simulation

\begin{tabular}{|r|r|r|r|r|r|r|r|r|r|r|}
\hline $\begin{array}{c}\text { No of } \\
\text { intersect } \\
\text { nodes / } \\
\text { Acti_pd }\end{array}$ & $\mathbf{1}$ & $\mathbf{2}$ & $\mathbf{3}$ & $\mathbf{4}$ & $\mathbf{5}$ & $\mathbf{6}$ & $\mathbf{7}$ & $\mathbf{8}$ & $\mathbf{9}$ & $\mathbf{1 0}$ \\
\hline $\mathbf{1}$ & 2 & 3 & 1 & 2 & 1 & 1 & 1 & 1 & 2 & 2 \\
\hline $\mathbf{2}$ & 1 & 1 & 1 & 1 & 1 & 1 & 1 & 1 & 1 & 1 \\
\hline $\mathbf{3}$ & 1 & 1 & 1 & 2 & 3 & 3 & 4 & 4 & 3 & 3 \\
\hline $\mathbf{4}$ & 1 & 1 & 1 & 1 & 1 & 1 & 2 & 3 & 3 & 2 \\
\hline $\mathbf{5}$ & 1 & 2 & 3 & 1 & 3 & 2 & 2 & 3 & 1 & 1 \\
\hline $\mathbf{6}$ & 1 & 1 & 1 & 1 & 1 & 1 & 3 & 3 & 1 & 1 \\
\hline $\mathbf{7}$ & 1 & 1 & 1 & 1 & 2 & 2 & 3 & 3 & 2 & 2 \\
\hline $\mathbf{8}$ & 1 & 1 & 1 & 1 & 1 & 1 & 2 & 4 & 2 & 3 \\
\hline $\mathbf{9}$ & 1 & 2 & 2 & 2 & 3 & 2 & 3 & 3 & 3 & 3 \\
\hline $\mathbf{1 0}$ & 1 & 1 & 1 & 1 & 1 & 1 & 3 & 2 & 2 & 2 \\
\hline $\mathbf{1 1}$ & 1 & 1 & 1 & 1 & 1 & 1 & 3 & 5 & 3 & 3 \\
\hline $\mathbf{1 2}$ & 1 & 1 & 1 & 1 & 1 & 1 & 1 & 1 & 3 & 2 \\
\hline $\mathbf{1 3}$ & 1 & 1 & 1 & 1 & 2 & 2 & 3 & 3 & 3 & 3 \\
\hline $\mathbf{1 4}$ & 1 & 1 & 1 & 1 & 2 & 2 & 2 & 2 & 2 & 3 \\
\hline $\mathbf{1 5}$ & 1 & 1 & 1 & 1 & 1 & 2 & 3 & 4 & 3 & 3 \\
\hline $\mathbf{1 6}$ & 1 & 1 & 1 & 1 & 1 & 1 & 3 & 2 & 2 & 2 \\
\hline $\mathbf{1 7}$ & 1 & 1 & 1 & 1 & 1 & 1 & 2 & 3 & 1 & 2 \\
\hline $\mathbf{1 8}$ & 1 & 1 & 1 & 2 & 3 & 2 & 2 & 3 & 2 & 3 \\
\hline $\mathbf{1 9}$ & 1 & 1 & 1 & 1 & 2 & 2 & 1 & 3 & 2 & 3 \\
\hline $\mathbf{2 0}$ & 1 & 1 & 1 & 1 & 1 & 1 & 2 & 3 & 3 & 2 \\
\hline & & & & & & & \\
\hline
\end{tabular}

\section{CONCLUSION}

The nodes are overlapped due to deployment randomly. The proposed protocols reduces the overlapping of the node by using radius and residual energy as a threshold of the overlapping for the connectivity amongs the nodes which are deployed. The proposed porotocol reduces the overlapping by $51.5 \%$ and the overlapped node can be used for increase the network life time using Sleep or Wakeup scheme. This increase the lifetime of the network by $50 \%$. Through table III shows that 1 indicates that they are connected but not overlapped 2, 3 and other shows that two nodes are overlapped, 3 nodes are overlapped and so on. These nodes are kept in Sleep \& Awake mode so that at any time only one can operate. So the life time is going to be increased around $50 \%$.

\section{REFERENCES}

1. Huang, Chi-Fu, and Yu-Chee Tseng. "The coverage problem in a wireless sensor network. Mobile networks and Applications" 10.4 (2005): 519-528.

2. Thai MT, Wang F, Du DH, Jia X. "Coverage problems in wireless sensor networks: designs and analysis". International Journal of Sensor Networks. 2008 May $1 ; 3(3): 191$.

3. Fan, GaoJun, and ShiYaoJin. "Coverage problem in wireless sensor network: A survey". Journal of networks 5.9 (2010): 1033 
4. S, SANGEETHA and RAMA LAKSHMI K. "A Survey on Coverage Problem in Wireless Sensor Network". International Journal of Advanced Research in Computer Engineering \&Technology ,Volume 1,Issue 10,December (2012).

5. Mini, S., Siba K. Udgata, and Samrat L. Sabat. $\square$ "Connected Coverage Problem in Wireless Sensor Networks".ISRN Sensor Networks 2012 (2012).

6. Chaturvedi, Pooja, and A. K. Daniel. "Recovery of holes problem in wireless sensor networks." International Conference on Information Communication and Embedded Systems (ICICES2014). IEEE, 2014.

7. Chaturvedi Pooja, Daniel AK. "Wireless sensor networks-a survey". In International conference on recent trends in information, telecommunication and computing 2014 (pp. 450-457).

8. Chaturvedi, Pooja, and Ajai K. Daniel. "Lifetime optimization for target coverage in wireless sensor networks." Proceedings of the 8th Annual ACM India Conference. ACM, 2015.

9. Chaturvedi, Pooja, and A. K. Daniel. "Performance analysis of characteristic parameters for coverage model in wireless sensor networks." 2016 3rd International Conference on Computing for Sustainable Global Development (INDIACom). IEEE, 2016.

10. Hirani, Priti K., and Manali Singh. "A survey on coverage problem in wireless sensor network",.International Journal of Computer Applications 116, no. 2 (2015).

11. Md.Amirul Islam,Rushdi Shams and Md.Zahirul Islam. "Minimization of Overlapping Coverage in Wireless Sensor Networks with Area-aware Coverage" (ICCCE 2010),11-13 May

12. Gungor VC, Lu B, Hancke GP." Opportunities and challenges of wireless sensor networks in smart grid". IEEE transactions on industrial electronics. 2010 Oct;57(10):3557-64.

13. Tarun Bala,Varsha Bhatia,Sunita Kumawat,Vivek Jaglan . "A survey :issues and challenges in Wireless sensor network". International Journal of Engineering \& Technology,7(2.4) (2018)53-55 